$2020,21(2)$, pp. 256 - 268, DOI: 10.18038/estubtda. 743369

\title{
ELECTRO-MECHANICAL IMPEDANCE BASED SHM OF THICK STRUCTURES IN BROAD-BAND FREQUENCY SPECTRUM
}

\author{
Mesut TEKKALMAZ ${ }^{1, *}$ \\ ${ }^{1}$ Mechanical Engineering Department, Faculty of Engineering and Architecture, Eskişehir Osmangazi University, \\ Eskişehir, Turkey
}

\begin{abstract}
In this study, theoretical and experimental studies were conducted in terms of electro-mechanical impedance spectroscopy (EMIS) to monitor the structural health status of thick metallic bar structures in broad-band frequency in the range up to 15 MHz. Thus, in-plane (longitudinal-wise) and out-of-plane (thickness-wise) mode EMI spectra were included in this range of frequency. Then, the thickness mode EMIS peaks were focused for pristine thick steel bar as a baseline and for bars with simulated damage in different depths and different locations. For theoretical part of the study, coupled field finite element analyses (CF-FEA) were carried out by modeling piezoelectric wafer active sensor (PWAS) bonded on steel bars in pristine and in damaged status. The thickness mode EMIS results from both CF-FEA simulations and EMIS measurements were analyzed to investigate the effects of the damage location and size variations through a statistical pattern recognition meth od as damage index such as the root mean square deviation, RMSD, method. It was found that RMSD values were similar trend for experimental and CF-FEA.
\end{abstract}

Keywords: Structural Health Monitoring (SHM), Electro-mechanical Impedance (EMI), Coupled Field Finite Element Analysis (CF-FEA), Damage detection

\section{INTRODUCTION}

Structural health monitoring (SHM) is a process in which specific strategies are applied to determine the damage's location / size and the remaining life of the structure after the damage has occurred. With SHM, the response of the structural system or components under varying loading and / or environmental conditions is continuously measured. SHM is used to monitor and assess the condition and abnormalities of structures and components in the working order during and after unexpected situations, due to damage [1].

SHM; observing the dynamic response of the system with the aid of sensors at regular intervals, and determining the current state of operation by means of statistical analyzes. In the long term, outputs of this process are regularly updated to provide information on whether or not the predefined functions of the structure have been fulfilled. After unexpected events, SHM is used for rapid status scanning and aims to provide reliable information about the integrity of the structure in real time [2]. Simultaneous perception of structural change in the SHM can be used to determine whether or not the structure is as designed.

The electromechanical impedance (EMI) technique is a relatively new technique for SHM. In the EMI technique, the lead-zirconate titanate piezoelectric ceramic sensor (PZT) patch is embedded or added in the structure to be monitored using a high-strength epoxy adhesive. The conductance characteristic of the patch is obtained in the high frequency range (30-400 kHz). This characteristic is a reference in assessing structural health. At any time when it is desired to evaluate the health of the structure, the conductance characteristic is re-acquired and compared with the reference conductance characteristic. The conductance characteristic of the bonded/embedded PZT patch is usually obtained by means of a

*Corresponding Author: tmesut@ogu.edu.tr

Received: 03.12.2018 Published: 15.06.2020 
commercially available impedance analyzer. The impedance analyzer implements an alternating voltage signal of $0.5 / 1$ Volt to the bonded/embedded PZT transducer over a predetermined frequency. The magnitude and phase of the steady-state current is recorded directly as the conductance and susceptance characteristic without any conversion [3].

There are many studies about the damage detection by EMI method in the literature. Rosiek et al. [4] investigated the notch damage at different depth $(1-4 \mathrm{~mm})$ by using EMI method experimentally and numerically by using the finite element method at the frequency range of $10-50 \mathrm{kHz}$. As a result of their experimental and numerical studies, they found that as the notch depth increased, resonance peaks shifted to the left and damage metric values increased. EMI admittance signals used in the diagnosis of structural damage. Hamzeloo et al. [5] have carried out experimental studies in order to detect different types and location of damage in different sizes of hollow cylinders made of aluminum and steel by using EMI method. They also proposed a finite element method. Experimental and numerical studies were performed at $10-40 \mathrm{kHz}$ frequency range. They found that the orientation, location and shape of the damage changed the value of RMSD damage metric, so it was difficult to correctly identify the damage. They also stated that the thickness and rigidity of the sample affected the results. Na and Lee [6] combined the EMI method with the artificial neural network method to detect the damaged area. They carried out experimental studies at $23-33 \mathrm{kHz}$ frequency range on composite plate. They found that $80 \%$ of the proposed method was correct. Wang et al. [7] presented a new method based on electromechanical admittance to detect defects in different locations and severities. They used CC as the damage metric. As a result of their experimental (30-50 kHz, 60-100 $\mathrm{kHz})$ and numerical $(30-50 \mathrm{kHz})$ studies, they suggested that proposed method was effective in determining the location and severity of the damage. Ai et al. [8] proposed a new EMI technique, combined with mechanical impedance (UMI), which uses the mechanical impedances of the PZT sensor and the structure. Then they examined the corrosion damage in a steel beam experimentally at $180-300 \mathrm{kHz}$ frequency range. As a result, they found that the recommended method for the detection of damage was more sensitive than the traditional method. Hu et al. [9] proposed an alternative damage metric to detect different defects. As a result of their experimental studies at $150-300 \mathrm{kHz}$ frequency range, they found that the metric they suggested yielded better than the RMSD damage metric for determining the damage location. da Silveira et al. [10] experimentally examined the different installation procedures (traditional, magnetic, metallic foil) of the PZT sensors using the EMI method. Experimental studies were carried out at $0-500 \mathrm{kHz}$ frequency band. They focused on the 20$30 \mathrm{kHz}, 50-60 \mathrm{kHz}$ and $120-130 \mathrm{kHz}$ 1sub-band. They used RMSD and CCDM as damage metrics. As a result, they concluded that the traditional method was more sensitive to damage detection. Tinoco et al. [11] presented a new approach for the EMI method that takes into account the electrical impedance of the free piezoelectric sensor. They carried out experimental studies at $10-45 \mathrm{kHz}$ frequency range. They concluded that the proposed model was successful for detecting the location of the damage. Liu et al. [12] used EMI method to determine freezing thawing and crack damage with PZT patches embedded in concrete blocks. Experimental studies were performed at $100-150 \mathrm{kHz}, 150-200 \mathrm{kHz}$, $200-250 \mathrm{kHz}, 250-300 \mathrm{kHz}, 300-400 \mathrm{kHz}, 400-500 \mathrm{kHz}$ frequency ranges. Freezing-thawing tests showed that resistance values increased as the number of cycles increased and peak values changed in decreasing frequency direction. They determined that as the depth of crack increases, there is a change in resistance signals. They also concluded that RMSD damage metric values increased as cycle and crack depth increased, and that the EMI method was more capable of detecting damage at relatively low frequencies. Ai et al. [13] proposed effective structural mechanical impedance (ESMI) method by using admittance values for damage detection in reinforced concrete structures. They performed experimental studies (180-300 kHz) for different defect levels using embedded PZT patches to verify the method. As a result, they suggested that the method can be an alternative method to detect structural damages by using embedded PZT patches in reinforced concrete structures.

In this study, defects in different lengths $(22,32,42 \mathrm{~mm})$ and depths $(0.5,1,1.5 \mathrm{~mm})$ were examined experimentally by using electromechanical impedance method (EMI). Simulation studies were conducted by using ANSYS finite element program. Experimental and numerical studies were 
performed at $30-80 \mathrm{kHz}$ and $30 \mathrm{kHz}-15 \mathrm{MHz}$ frequency ranges. It is interesting to note that experimental and numerical study focusing on high frequencies was no dealt with in details. When similar studies in the literature are examined, no experimental and numerical study was performed at such high frequencies.

\section{THEORY of THE EMI METHOD}

The impedance-based SHM technique is performed by using electromechanical properties of a coupling effect between the PWAS patch and the host structure. Using the 1-D electromechanical system, the binding effect of a system with a PWAS and a host structure and electro-mechanical impedance is shown in Figure 1. The PWAS is powered with alternating voltage and changed their frequency characteristics of electrical impedance accordingly to fluctuations of mechanical properties of monitored structure.

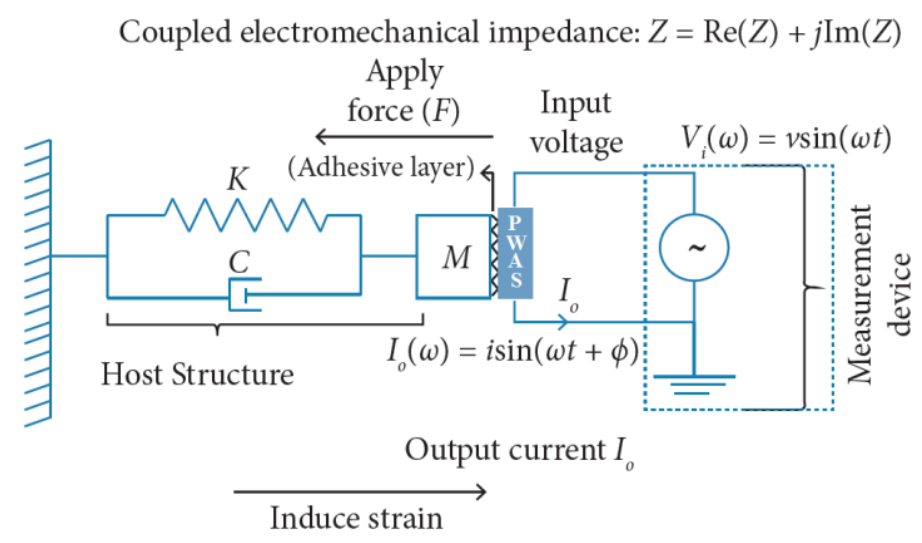

Figure 1. One-Dimensional EMI system.

Any changes in the structure, such as damage, lead to changes in the mechanical impedance of the structure affecting the PWAS electrical impedance by the electromechanical coupling effect of PWAS. The mechanical impedance of PWAS in Figure 1 is defined as the ratio of a harmonic input voltage to current at an angular frequency in the frequency domain. Similarly, the mechanical impedance of the material is defined as the ratio of a harmonic excitation force at an angular frequency to the velocity response in the frequency domain. The complex electrical impedance can be expressed as:

$$
Z(\omega)=\left[j \omega \frac{w l}{s}\left(\frac{d_{31}^{2} Y_{11}^{E} Z_{P}(\omega)}{Z_{P}(\omega)+Z_{S}(\omega)} \frac{\tan (\kappa l)}{\kappa l}+\varepsilon_{33}^{T}-d_{31}^{2} Y_{11}^{E}\right)\right]^{-1}
$$

where $Z_{P}$ is the impedance of the PWAS patch, and $Z_{S}$ is the loading impedance of the host structure obtained as

$$
\begin{gathered}
Z_{P}(\omega)=\frac{V(\omega)}{I(\omega)}=\frac{\kappa w s Y_{x x}^{E}}{j \omega \tan (\kappa l)} \\
Z_{S}(\omega)=\frac{F_{0}(\omega)}{\dot{x}_{0}(\omega)}=c+j\left(\frac{m \omega^{2}-k}{\omega}\right)
\end{gathered}
$$

where $\kappa$ is a wave number $\left(\omega / c_{t}^{E}\right), \omega$ is an excitation frequency, $c_{t}^{E}$ is a wave velocity $\left(\sqrt{Y_{x x}^{E} / \rho}\right)$ $\rho$ is density, $Y_{x x}^{E}$ is an elastic stiffness at a constant electric field, $d_{3 x}$ is the piezoelectric strain coefficient, $\varepsilon_{33}^{T}$ is a permittivity at constant stress; $l, w$ and $s$ are the length, width and thickness of the PWAS patch respectively, $F_{0}$ is a resultant force, $\dot{x}_{0}$ is the amplitude of an instantaneous velocity response, and $m, c$ and $k$ are mass, damping and stiffness of the host structure, $j=\sqrt{-1}$. 
The evaluation of EMI measurements for SHM is generally carried out in two steps. First, the pristine structure is identified by its frequency response represented by the electrical impedance $Z^{0}$ at the PWAS transducer. This measurement is called base line measurement. Second, the electrical impedance $Z$ of the considered structure is continuously monitored. Changes to the base line measurement are evaluated for damage identification. Typical approaches for the evaluation are, e.g., damage metrics, spectral features, statistical approaches, pattern recognition methods and neural networks [14-15]. In this work, the root mean square deviation (RMSD) is used as the damage metric approach. Damage metrics provide a measure - the damage index - for the deviation of a structure dynamic frequency response - represented by $\operatorname{Re}\left(Z_{i}\right)$ - and a base line measurement - represented by $\operatorname{Re}\left(Z_{i}^{0}\right) . N$ is the number of single measurements at different frequencies within the considered frequency range.

$$
\operatorname{RMSD}(\%)=\left(\sum_{i=1}^{N}\left[\operatorname{Re}\left(Z_{i}\right)-\operatorname{Re}\left(Z_{i}^{0}\right)\right]^{2} / \sum_{i=1}^{N}\left[\operatorname{Re}\left(Z_{i}^{0}\right)\right]^{2}\right)^{1 / 2}
$$

\section{EXPERIMENTAL SETUP}

The measurement of the EMIS was performed with Keysight E4990A Impedance Analyzer. For PWAS EMIS test, two probes are connected to the PWAS and the specimen to measure the EMIS of the PWAS coupled with the host structure such as undamaged steel bars and damaged steel bars as shown in Figure 2.

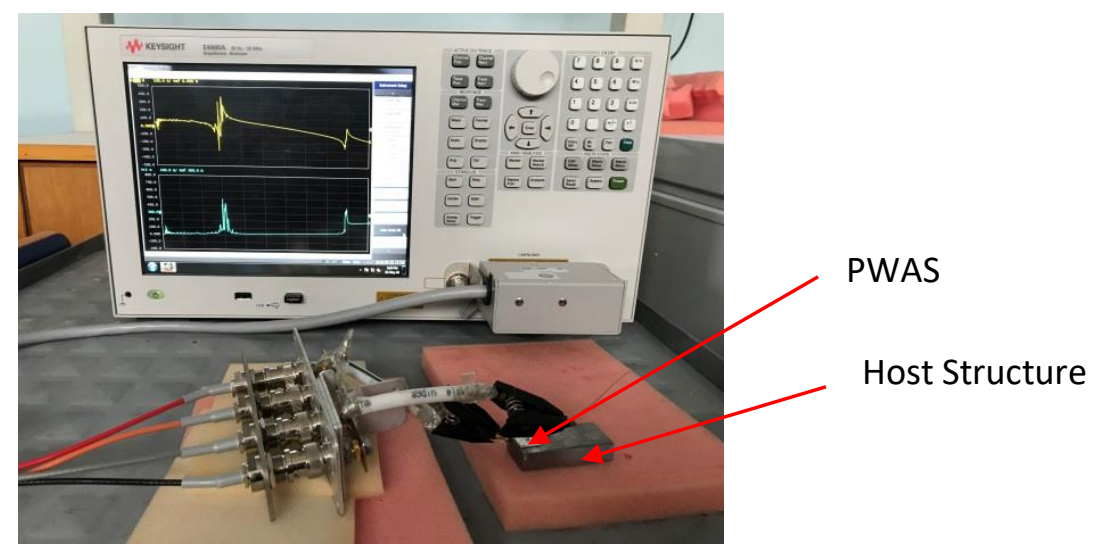

Figure 2. Keysight E4990A Impedance Analyzer and PWAS-EMIS experimental setup.

A thick steel bar was tested for the detection of progressive and depth of changing damage, as shown in Figure 2. The $10 \mathrm{~mm} \times 10 \mathrm{~mm} \times 0.5 \mathrm{~mm}$ square PWAS resonators are bonded to the $50 \mathrm{~mm} \times 20$ $\mathrm{mm} \times 10 \mathrm{~mm}$ size steel bar using the adhesive glue on the left edge, as shown in Figure 3. The depthvarying surface damage was also investigated with artificial damages at a depth of $0.5 \mathrm{~mm}, 1 \mathrm{~mm}$ and $1.5 \mathrm{~mm}$, and a length of $\mathrm{A}=22 \mathrm{~mm}$, respectively. The progressive surface damage on the steel bar was simulated as artificial damages at regular intervals of $22 \mathrm{~mm}, 32 \mathrm{~mm}$ and $42 \mathrm{~mm}$ and at a depth of $\mathrm{B}=1.5 \mathrm{~mm}$. Damaged situations were listed in Table 1 . The PWAS samples were excited about $0.5 \mathrm{~V}$ and scanned over a predetermined frequency range of low frequency band $(30 \mathrm{kHz}-80 \mathrm{kHz})$ and high frequency band $(30 \mathrm{kHz}-15 \mathrm{MHz})$ and recorded in terms of the complex impedance control the impedance analyzer and sweep the frequency range discretizing by 1000 steps. 


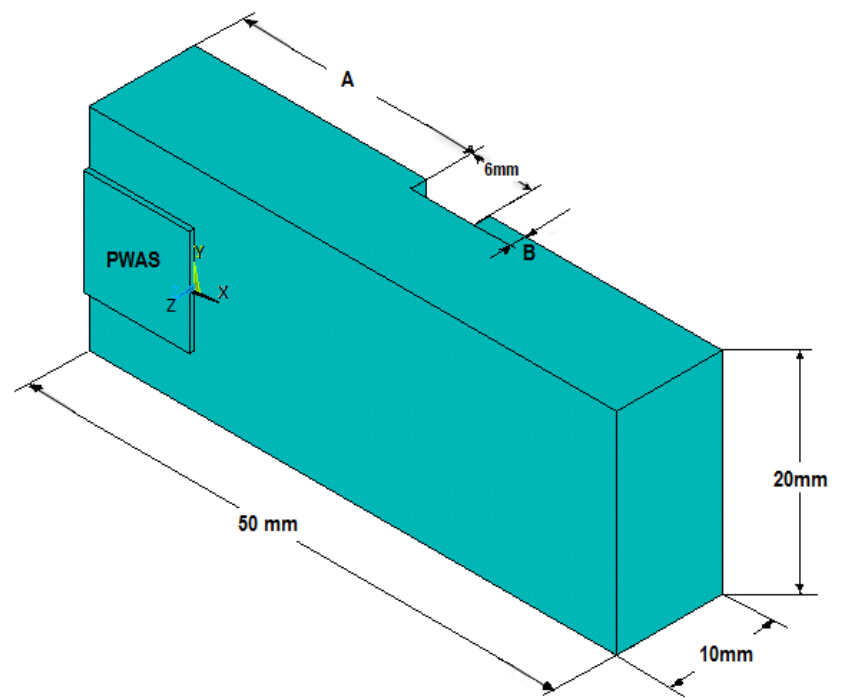

Figure 3. Experimental setup and PWAS resonator bonded on steel bar.

Table 1. Surface damage location.

\begin{tabular}{ccc}
\hline Damage Location & & \\
\hline Case 1 & $\mathrm{~A}=22 \mathrm{~mm}$ & $\mathrm{~B}=0.5 \mathrm{~mm}$ \\
Case 2 & $\mathrm{~A}=22 \mathrm{~mm}$ & $\mathrm{~B}=1.0 \mathrm{~mm}$ \\
Case 3 & $\mathrm{~A}=22 \mathrm{~mm}$ & $\mathrm{~B}=1.5 \mathrm{~mm}$ \\
Case 4 & $\mathrm{~A}=32 \mathrm{~mm}$ & $\mathrm{~B}=1.5 \mathrm{~mm}$ \\
Case 5 & $\mathrm{~A}=42 \mathrm{~mm}$ & $\mathrm{~B}=1.5 \mathrm{~mm}$ \\
\hline
\end{tabular}

\section{COUPLED FIELD FINITE ELEMENT ANALYSES}

In this section, ANSYS multi-physics software was used to model the structural system and to compute the electro-mechanical impedance of the system. The CF-FEA model handles the interaction between structural and electrical fields. The CF-FEA model was used to evaluate the effects of damage changes on the impedance spectra of the PWAS bonded on a steel bar with progressive and varying damage depth. The PWAS was modeled using SOLID5 element which is a three-dimensional coupled field solid element with eight nodes and six degrees of freedoms (DOF) at each node. This element has an electrical voltage used as an additional DOF. In order to model the steel bar, a SOLID45 element was used. This element is defined by eight nodes and 3 DOF at each node, with translations in the nodal $\mathrm{x}, \mathrm{y}$, and $\mathrm{z}$ directions. The 3D CF-FEA model was shown in Figure 4.

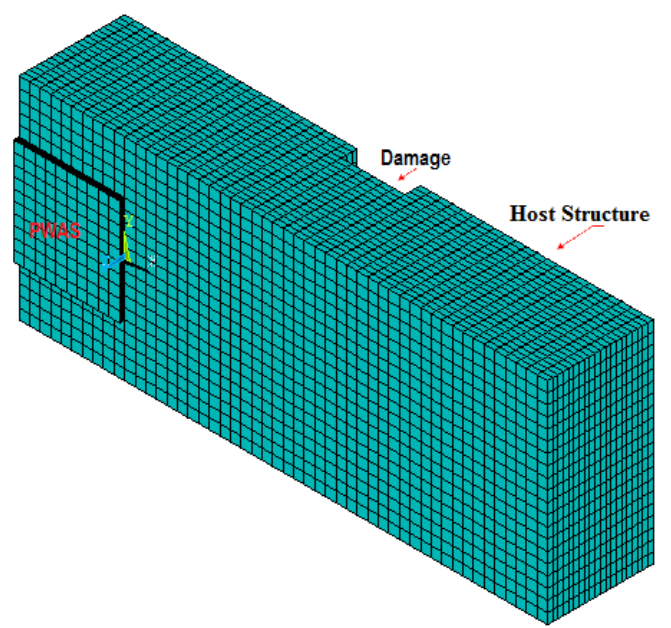

Figure 4. CF-FEA model for PWAS bonded on steel bar. 
A square shaped PWAS in dimensions of $10 \mathrm{~mm} \times 10 \mathrm{~mm} \times 0.5 \mathrm{~mm}$ was modeled with the steel substrate in dimensions of $50 \mathrm{~mm} \times 20 \mathrm{~mm} \times 10 \mathrm{~mm}$. The PIC- 255 material properties provided by PI Ceramic, Inc. catalog were assigned to the PWAS as follows. The catalog data for the piezoelectric material is also presented in Table 2 .

The three kinds of material properties for CF-FEA were required: and stiffness matrix $\left[C_{p}\right]$, which is a $6 x 6$ symmetric matrix, permittivity matrix $\left[\varepsilon_{p}\right]$; piezoelectric matrix $\left[e_{p}\right]$, which is a $6 x 3$ matrix that relates the electrical field to stress; These properties were assumed as follows:

$$
\begin{gathered}
{\left[C_{p}\right]=\left[\begin{array}{cccccc}
108.8 & 62.4 & 58.2 & 0 & 0 & 0 \\
62.4 & 108.8 & 58.2 & 0 & 0 & 0 \\
58.2 & 58.2 & 87.9 & 0 & 0 & 0 \\
0 & 0 & 0 & 23.2 & 0 & 0 \\
0 & 0 & 0 & 0 & 18 & 0 \\
0 & 0 & 0 & 0 & 0 & 18
\end{array}\right][\mathrm{GPa}]} \\
{\left[\varepsilon_{p}\right]=\left[\begin{array}{cccc}
1033.86 & 0 & 0 \\
0 & 1033.86 & 0 \\
0 & 0 & 801.69
\end{array}\right] \times 10^{-8}[\mathrm{~F} / \mathrm{m}]} \\
{\left[e_{P}\right]=\left[\begin{array}{cccc}
0 & 0 & -7.53 \\
0 & 0 & -7.53 \\
0 & 0 & 14.2 \\
0 & 0 & 0 \\
0 & 9.91 & 0 \\
9.91 & 0 & 0
\end{array}\right]\left[\mathrm{C} / \mathrm{m}^{2}\right]}
\end{gathered}
$$

The density of the PWAS material is assumed to be $\rho=7800 \mathrm{~kg} / \mathrm{m}^{3}$. The material properties of the structural steel were assigned as follows: the Elasticity modulus is $210 \mathrm{GPa}$, the density is $7800 \mathrm{~kg} / \mathrm{m}^{3}$ and the Poisson ratio is assumed to be 0.29 .

Table 2. Properties of piezoelectric PIC255 material.

\begin{tabular}{lcc}
\hline & Value & Unit \\
\hline Density $\rho$ & 7800 & $\mathrm{~kg} / \mathrm{m}^{3}$ \\
Poisson's ratio $v$ & 0.34 & - \\
Relative permittivity in the polarization $\varepsilon_{33}^{T}$ & $1750 \varepsilon_{0}$ & - \\
Relative permittivity direction $\perp$ to polarization $\varepsilon_{11}^{T}, \varepsilon_{22}^{T}$ & $1650 \varepsilon_{0}$ & - \\
Elastic compliance coefficient $S_{11}^{E}, S_{22}^{E}$ & 16.1 & $10^{-12} \mathrm{~m}^{2} / \mathrm{N}$ \\
Elastic compliance coefficient $S_{33}^{E}$ & 20.7 & $10^{-12} \mathrm{~m}^{2} / \mathrm{N}$ \\
Dielectric loss factor $\delta$ & 0.02 & - \\
Coupling factor for transverse oscillation $k_{31}$ & 0.35 & - \\
Transverse piezoelectric large-signal deformation coefficient $d_{31}, d_{32}$ & -180 & $10^{-12} \mathrm{C} / \mathrm{N}$ \\
Longitudinal piezoelectric large-signal deformation coefficient $d_{33}$ & 400 & $10^{-12} \mathrm{C} / \mathrm{N}$ \\
Shear deformation coefficient $d_{15}, d_{16}$ & 500 & $10^{-12} \mathrm{C} / \mathrm{N}$ \\
\hline
\end{tabular}

Note: $\varepsilon_{0}=8.85 \times 10^{-12} \mathrm{~F} / \mathrm{m}$ is the permittivity of free space. 


\section{RESULTS AND DISCUSSION}

\subsection{Experimental Results}

Experimental studies carried out undamaged and damaged steel bars at $30-80 \mathrm{kHz}$ and $30 \mathrm{kHz}-15$ $\mathrm{MHz}$ frequency ranges. Experimental results at $30-80 \mathrm{kHz}$ frequency range were plotted in Figure 5.

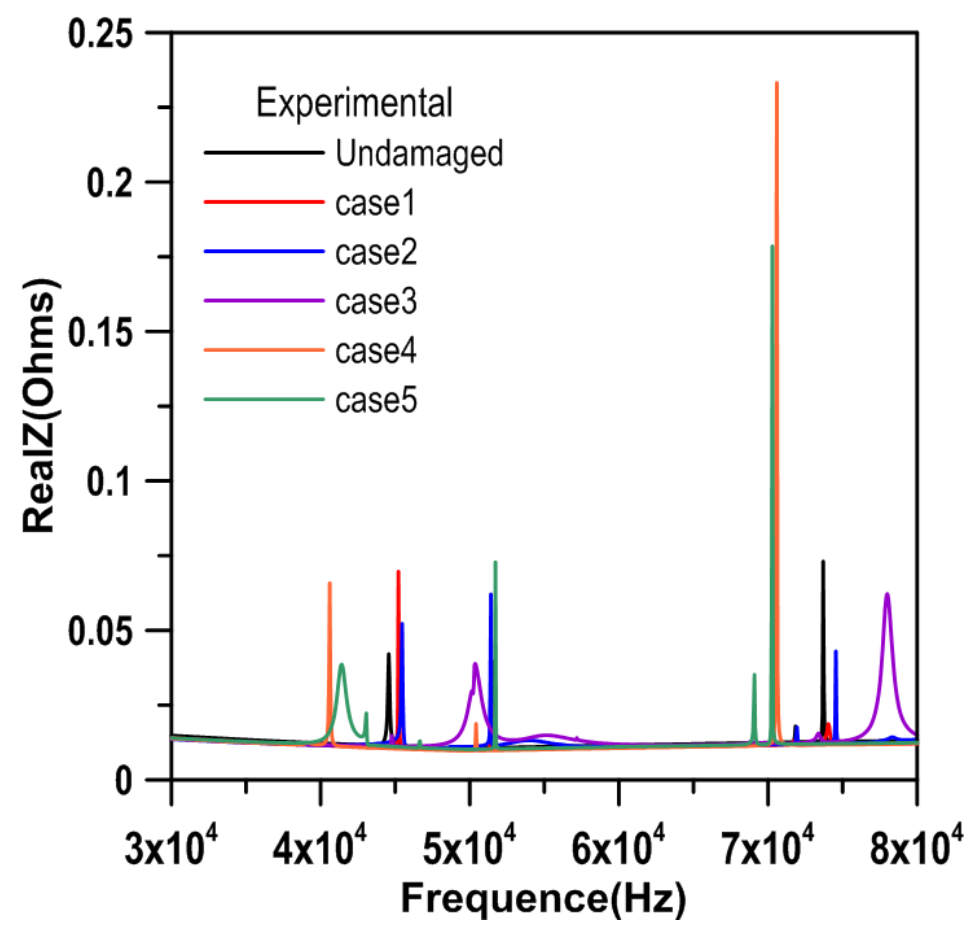

Figure 5. Experimental results $(30-80 \mathrm{kHz})$

In Figure 5, it was clear that the resonance peaks were in the $40-55 \mathrm{kHz}$ and $70-80 \mathrm{kHz}$ frequency range. Although the change in resonance peaks can't be clearly seen, a change was observed as the damage situation changes. Therefore, the frequency and amplitude values of the resonance peaks were given in Table 3.

Table 3. Frequency and real impedance values at resonance peaks

\begin{tabular}{ccc}
\hline & Frequency $(\mathrm{Hz})$ & Real impedance $($ Ohm $)$ \\
\hline \multirow{2}{*}{ Undamaged } & 44564.56 & 0.042 \\
& 73693.69 & 0.073 \\
Case 1 & 45215.22 & 0.070 \\
& 74044.04 & 0.019 \\
Case 2 & 51421.42 & 0.062 \\
& 74544.54 & 0.043 \\
Case 3 & 50370.37 & 0.038 \\
& 77998.00 & 0.062 \\
Case 4 & 40610.61 & 0.066 \\
& 70590.59 & 0.233 \\
Case 5 & 51721.72 & 0.073 \\
& 70290.29 & 0.179 \\
\hline
\end{tabular}

When the experimental results were examined in the resonance peaks, it was observed that frequency values increase generally for $40-55 \mathrm{kHz}$ and increase for $70-80 \mathrm{kHz}$ as damage depth increases. For 
amplitude values, no clear trend was observed. When damage length increased, frequency values generally increased for $40-55 \mathrm{kHz}$ and generally decreased for $70-80 \mathrm{kHz}$. For amplitude values, no clear trend was observed. Also new resonance peaks were observed for case 3 and 5. This was due to the increased complexity of the structure. These results showed that it was difficult to establish a definite trend between damage and frequency.

After the experiments in the frequency range of $30-80 \mathrm{kHz}$ were completed, experiments were performed in the frequency range of $30 \mathrm{kHz}-15 \mathrm{MHz}$ with the same experimental procedures. Experimental results at $30 \mathrm{kHz}-15 \mathrm{MHz}$ frequency range were plotted in Figure 6. In Figure 6, it was clear that the maximum resonance peaks were in the 4-6 $\mathrm{MHz}$ frequency range. As the damage condition changed, the change in resonance peaks were clearly seen in Figure 6. Frequency and amplitude values of the resonance peaks were given in Table 4.

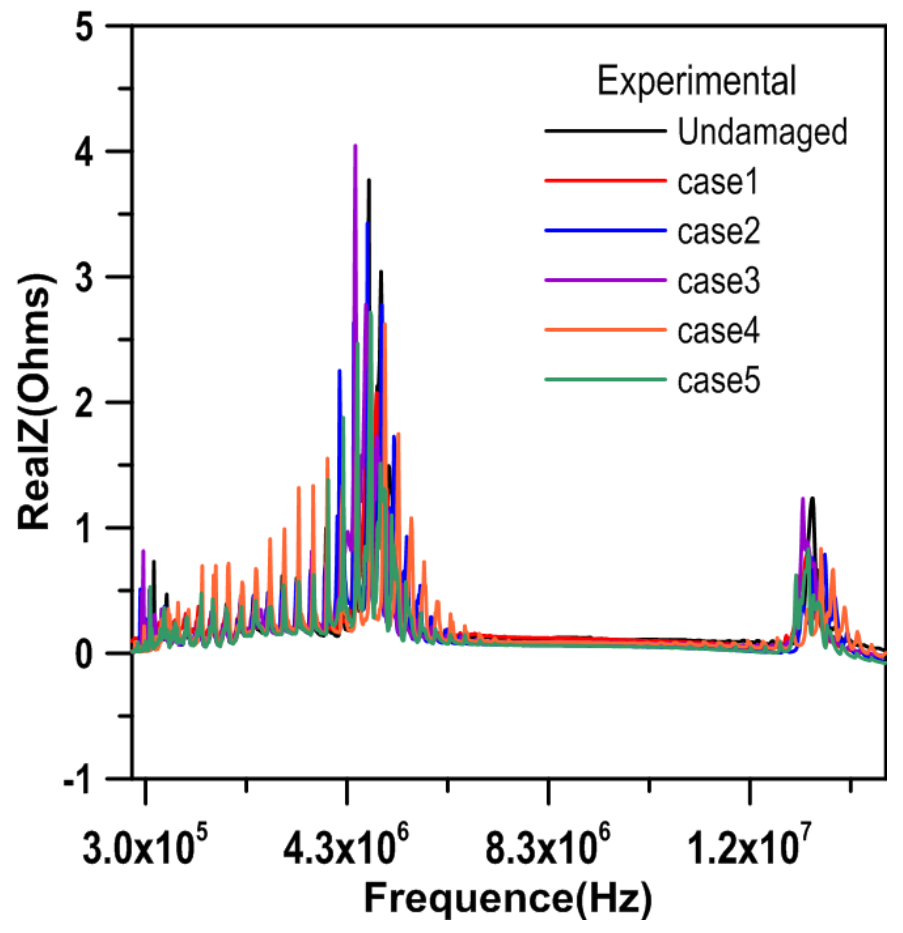

Figure 6. Experimental results (30 kHz-15 MHz).

Table 4. Frequency and real impedance values at resonance peaks

\begin{tabular}{ccc}
\hline & Frequency $(\mathrm{Hz})$ & Real impedance $($ Ohm $)$ \\
\hline \multirow{2}{*}{ Undamaged } & 4735285 & 3.772 \\
& 13546456 & 1.237 \\
Case 1 & 4705315 & 2.657 \\
& 13426577 & 0.774 \\
Case 2 & 4705315 & 3.427 \\
& 13786216 & 0.787 \\
Case 3 & 4465556 & 4.045 \\
& 13351652 & 1.232 \\
Case 4 & 5049970 & 2.625 \\
& 13711291 & 0.834 \\
Case 5 & 4765255 & 2.717 \\
\end{tabular}


When the experimental results in the high frequency range were examined, it was seen that the frequency values in the resonance peaks generally decreased as the depth of the damage increased. For amplitude values, no clear trend was observed. As the damage length increased, the frequency values in the resonance peaks were not clear trend. Amplitude values generally decreased as the damage length increased.

\subsection{CF-FEA Results}

Numerical studies carried out, using finite element program ANSYS ${ }^{\circledR}$, undamaged and damaged bars at $30-80 \mathrm{kHz}$ and $30 \mathrm{kHz}-15 \mathrm{MHz}$ frequency ranges. Damaged situations were listed in Table 1. Numerical (CF-FEA) results at 30-80 kHz frequency range were plotted in Figure 7.

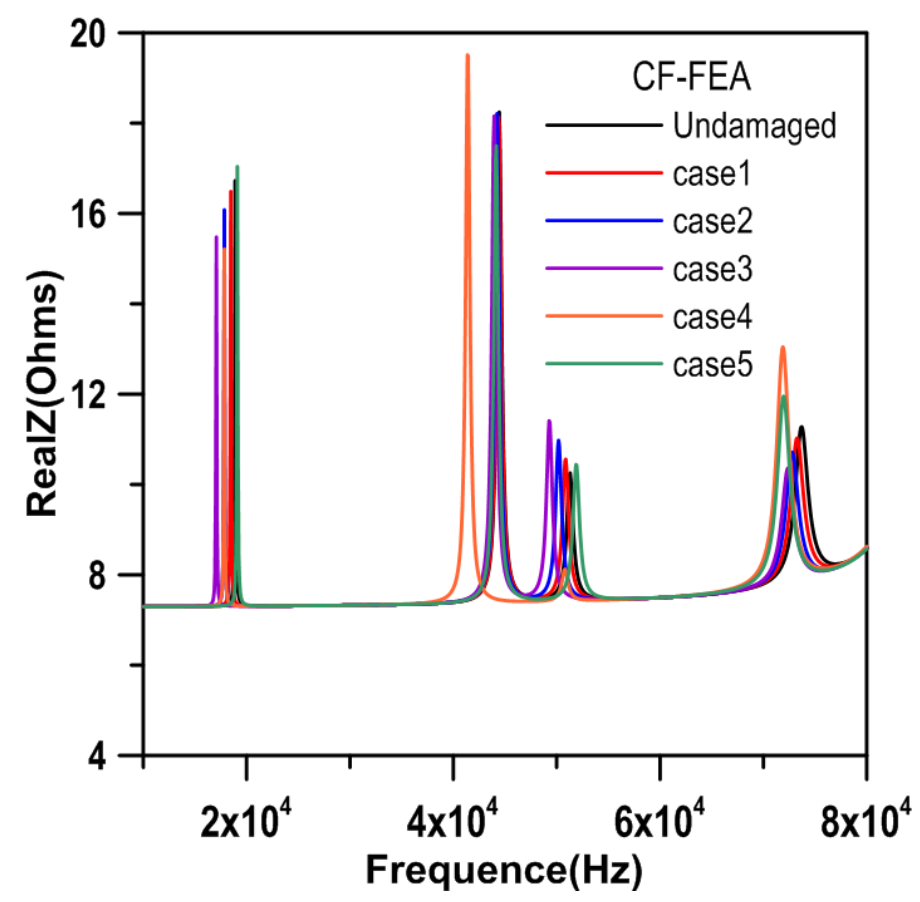

Figure 7. CF-FEA results $(30-80 \mathrm{kHz})$

In Figure 7, it was clear that the resonance peaks were in the $40-55 \mathrm{kHz}$ and $70-80 \mathrm{kHz}$ frequency range. It was similar trend with experimental results. Frequency and amplitude values of the resonance peaks were given in Table 5 .

Table 5. Frequency and real impedance values at resonance peaks

\begin{tabular}{ccc}
\hline & Frequency $(\mathrm{Hz})$ & Real impedance $(\mathrm{Ohm})$ \\
\hline Undamaged & 44440 & 18.24 \\
& 73700 & 11.28 \\
Case 1 & 44370 & 18.13 \\
& 73280 & 11.02 \\
Case 2 & 44230 & 18.21 \\
& 72790 & 10.71 \\
Case 3 & 43950 & 18.16 \\
& 72370 & 10.37 \\
Case 4 & 41360 & 19.51 \\
& 71880 & 13.05 \\
Case 5 & 44160 & 17.50 \\
& 71950 & 11.96 \\
\hline
\end{tabular}


When the CF-FEA results were examined in the resonance peaks, it was observed that frequency values decreased generally for $40-55 \mathrm{kHz}$ and decreased for $70-80 \mathrm{kHz}$ as damage depth increased. For amplitude values; although no clear trend was observed at $40-55 \mathrm{kHz}$, always decreased at 70-80 $\mathrm{kHz}$ frequency range as damage depth increased. When damage length increased, frequency values generally decreased for $40-55 \mathrm{kHz}$ and $70-80 \mathrm{kHz}$. For amplitude values, no clear trend was observed.

In summary, as the depth of the damage increased in the $70-80 \mathrm{kHz}$ frequency range, the frequency and amplitude values of the resonance peaks were continuously reduced. Although the frequency values of the resonance peaks were generally in a decreasing trend, it was difficult to detect a clear relationship between the frequency change and the different damage types. It was seen that experimental and CF-FEA results were similar trend $70-80 \mathrm{kHz}$ frequency range.

After the numerical studies in the frequency range of $30-80 \mathrm{kHz}$ were completed, numerical studies were performed in the frequency range of $30 \mathrm{kHz}-15 \mathrm{MHz}$ with the same finite element procedures. CF-FEA results at $30 \mathrm{kHz}-15 \mathrm{MHz}$ frequency range were plotted in Figure 8. As the damage condition changed, the change in resonance peaks were not clearly seen in Figure 8. For this reason, frequency and amplitude values of the resonance peaks were given in Table 6.

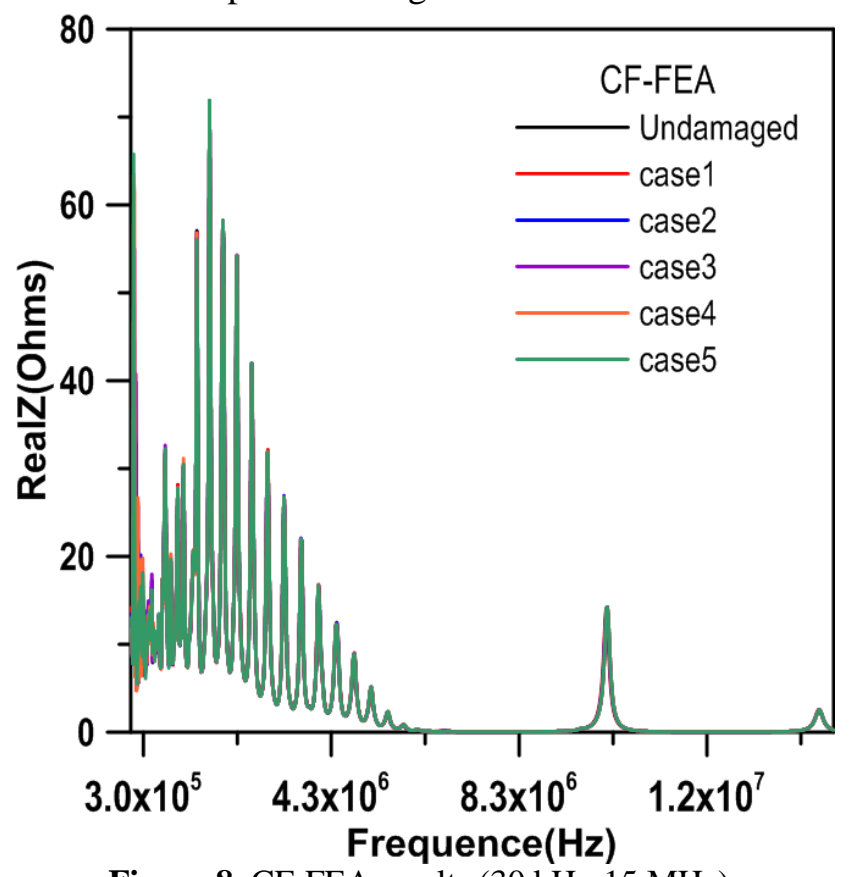

Figure 8. CF-FEA results (30 kHz-15 MHz).

Table6. Frequency and real impedance values at resonance peaks

\begin{tabular}{ccc}
\hline & Frequency $(\mathrm{Hz})$ & Real impedance $(\mathrm{Ohm})$ \\
\hline \multirow{2}{*}{ Undamaged } & 1706640 & 71.86 \\
& 10179660 & 14.25 \\
Case 1 & 1706640 & 70.90 \\
& 10149720 & 13.92 \\
Case 2 & 1706640 & 71.30 \\
& 10164690 & 14.20 \\
Case 3 & 1706640 & 71.52 \\
& 10179660 & 14.25 \\
Case 4 & 1706640 & 71.73 \\
& 10179660 & 14.25 \\
Case 5 & 1706640 & 71.91 \\
& 10179660 & 14.25 \\
\hline
\end{tabular}


Tekkalmaz, Eskişehir Technical Univ. J. of Sci. and Tech. A-Appl. Sci. and Eng. 21 (2)-2020

When the CF-FEA results in the high frequency range were examined, it was seen that there were no frequency change at $1-3 \mathrm{MHz}$ as the depth of the damage increased. Also the frequency values in the resonance peaks $(9-11 \mathrm{MHz})$ generally decreased as the depth of the damage increased. When the damage length increased, the frequency values in the resonance peaks were not changed. For amplitude values, no clear trend was observed as the length and depth of the damage increased.

In both the low frequency range and the high frequency range, RMSD values were considered to be indicators of damage and the results were shown in Figures 9 and 10, respectively.

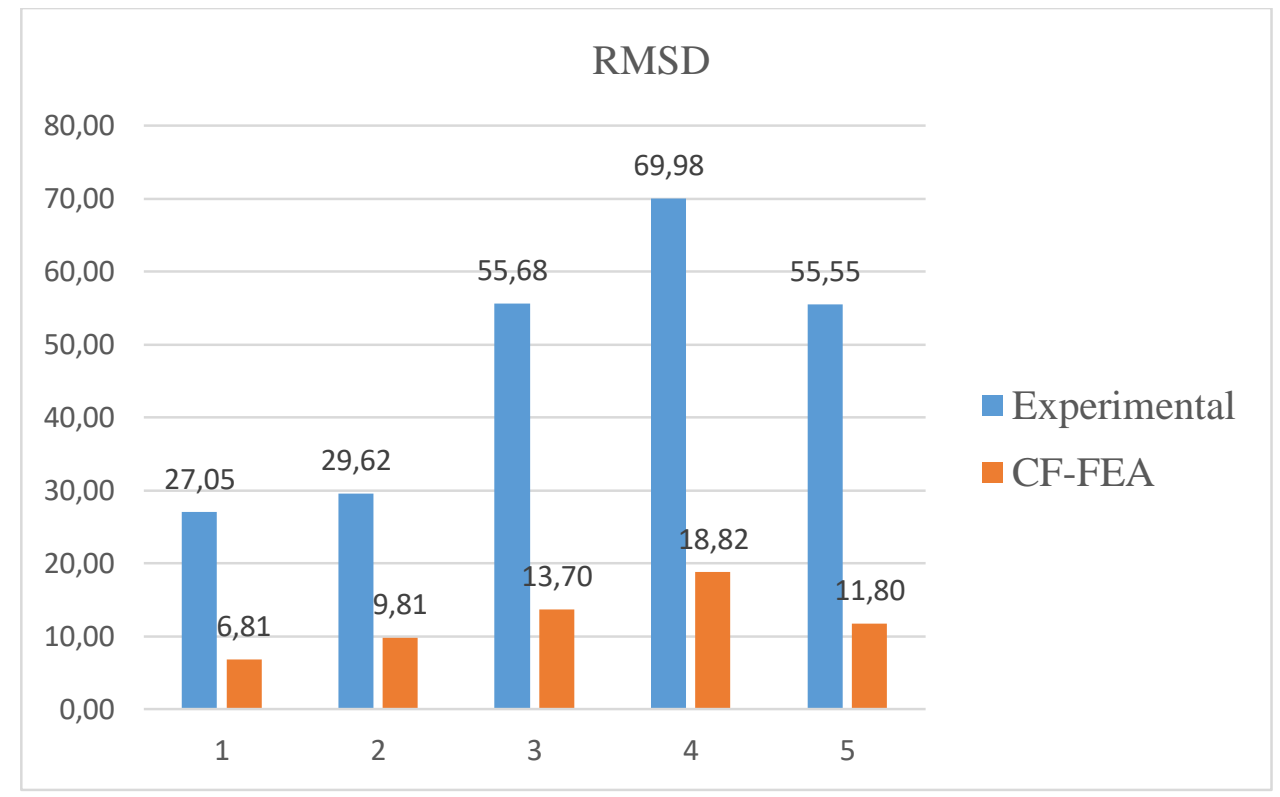

Figure 9. RMSD values for different damage cases $(30-80 \mathrm{kHz})$.

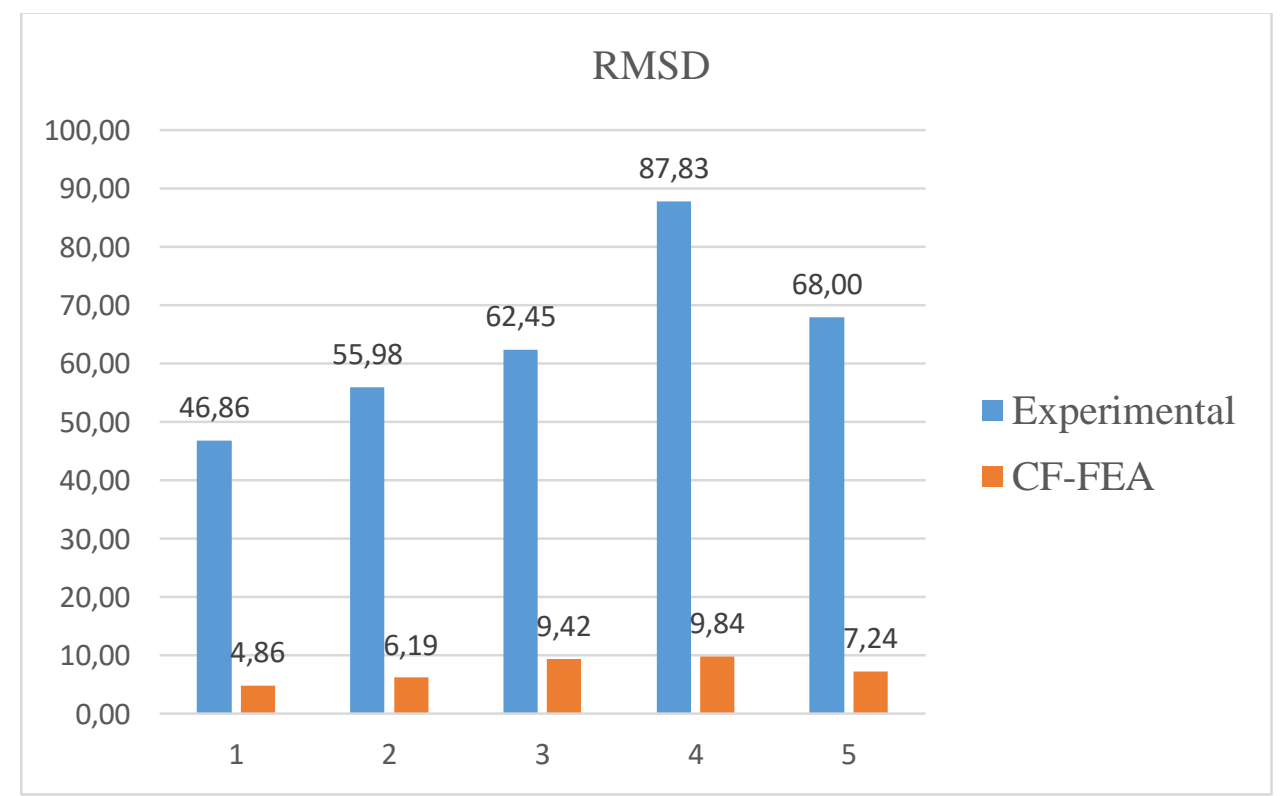

Figure 10. RMSD values for different damage cases $(30 \mathrm{kHz}-15 \mathrm{MHz})$.

For both the low frequency range and the high frequency range, the damage indicators showed similar changes. The high frequency RMSD values in the experimental results determine the depth of the 
damage also gave more pronounced results. Both the low frequency and high frequency RMSD values showed similar changes in locating the damage. Low frequency RMSD deviations in CF-FEA were more pronounced than high frequency RMSD values. As the damage depth increased, the RMSD values were increased. As the damage length increased, the RMSD values were firstly increased then decreased both experimental and CF-FEA results. These results confirmed the reality of the experimental results very well. In additionally, the results were found to be consistent with the studies conducted at low frequencies in the literature $[5,7,9,12,13]$.

\section{CONCLUSION}

In this study, experimental and numerical studies were performed at low and high frequency ranges for the detection of different defects. It was found that low and high frequency analysis results similar to each other. Experimental and CF-FEA results were more compatible with low frequencies. It was seen that there were more resonance peak at high frequencies.

In the frequency domain, it was determined that experimental and CF-FEA results showed the same trend at $70-80 \mathrm{kHz}$ frequency range as the damage depth and length increased. In addition, it was determined that experimental and CF-FEA results showed the same trend at 9-15 $\mathrm{MHz}$ as damage depth increased.

As the damage depth increased, the RMSD values were increased. As the damage length increased, the RMSD values were firstly increased then decreased for both experimental and CF-FEA results. It was also found that RMSD values were similar trend for experimental and CF-FEA.

The results showed that the studies performed at high frequencies can be used for damage detection. In addition, the results of CF-FEA showed similar results with experimental results. In the absence of experimental study; CF-FEA studies, especially low frequencies $(70-80 \mathrm{kHz})$ and high frequencies (9$15 \mathrm{MHz}$ ), were found to yield successful results.

In future studies, the size effect can be investigated by changing the size of the sample. In addition, model improvements can be carried out to bring CF-FEA results closer to experimental results at higher frequencies. The alternative damage metric can be improved and the ability of the method to detect defects can be better evaluated.

\section{REFERENCES}

[1] Aktan A, Helmicki A., Hunt V. Issues in health monitoring for intelligent infrastructure, Smart Mater Struct 1998; 7: 674-692.

[2] Doebling SW, Farrar CR, Prime MB. A summary review of vibration-based damage identification methods, Shock and Vibration Digest 1998: 30, 91-105.

[3] Shankar, R. An integrated approach for Structural health monitoring, $\mathrm{PhD}$, Indian Institute of Technology, Delhi, India, 2009.

[4] Rosiek M, Martowicz A, Uhl T, Stępiński T, Łukomski T. Electromechanical impedance method for damage detection in mechanical structures, 2010, Proceedings of 11th IMEKO TC, 10, 18-20.

[5] Hamzeloo S R, Shamshirsaz M, Rezaei SM. Damage detection on hollow cylinders by electromechanical impedance method: Experiments and finite element modeling, CR Mecanique 2012: $340,668-677$. 
[6] Na S, Lee HK. Neural network approach for damaged area location prediction of a composite plate using electromechanical impedance technique, Compos Sci Technol 2013:88, 62-68.

[7] Wang D, Song H, Zhu H. Numerical and experimental studies on damage detection of a concrete beam based on PZT admittances and correlation coefficient, Constr Build Mater 2013; 49, 564574.

[8] Ai D, Zhu H, Luo H, Yang J. An effective electromechanical impedance technique for steel structural health monitoring, Constr Build Mater 2014; 73, 97-104.

[9] $\mathrm{Hu} \mathrm{X}$, Zhu H, Wang D. A study of concrete slab damage detection based on the electromechanical impedance method, Sensors 2014; 14, 19897-19909.

[10] da Silveira R Z, Campeiro L M, Baptista F G. Analysis of Sensor Installation Methods in Impedance-based SHM Applications, Procedia Engineer 2016; 168, 1751-1754.

[11] Tinoco H A, Robledo-Callejas L, Marulanda D J, Serpa AL. Damage detection in plates using the electromechanical impedance technique based on decoupled measurements of piezoelectric transducers, J Sound Vib 2016; 384, 146-162.

[12] Liu, P, Wang W, Chen Y, Feng X, Miao L, Concrete damage diagnosis using electromechanical impedance technique, Constr Build Mater 2017; 136, 450-455.

[13] Ai D, Zhu H, Luo H, Wang C. Mechanical impedance based embedded piezoelectric transducer for reinforced concrete structural impact damage detection: A comparative study, Constr Build Mater 2018; 165, 472-483.

[14] Giurgiutiu V, Zagrai AN, Damage detection in thin plates and aerospace structures with the electro-mechanical impedance method, Struct Health Monit 2005; 4, 99-118.

[15] Giurgiutiu V, Structural Health Monitoring with Piezoelectric Wafer Active Sensors, Academic Press Inc., 2014. 